\title{
ARHGAP21 modulates the secretion of VLDL vesicles in the liver of C57BL/6 mice
}

\author{
Lucas Zangerolamo*, Gabriela M. Soares, Sara T. Saad, Antonio Carlos Boschero, Helena C. L. Barbosa- \\ Sampaio
}

\begin{abstract}
ARHGAP21 controls multiple cellular functions, such as migration, proliferation, differentiation and vesicles intracellular traffic. Our ARHGAP21-heterozygous animal model (Het, $50 \%$ of the protein expression) treated with the high fat diet (HFD) have higher content of triglycerides in the liver, compared to C57BL/6 animals fed on the same diet. The HFD favored the accumulation of lipids in the Het animal. The liver of our model fed with HFD is more sensitive to insulin than the control animal, which may have led to increased degradation of the proteins involved in the assembly of VLDL, such as MTP, preventing this molecule from being secreted and consequently has became the animal's liver steatotic. In addition, there is a greater gene expression of GLUT2 in het animals, this more glucose reaches the hepatocytes, this overload of glucose may be converted in lipids, and it collaborates with the steatotic state found in this animal.
\end{abstract}

Key words: hepatic steatosis, ARHGAP21, high fat diet

\section{Introduction}

ARHGAP21 is a RhoGAP involved in several cellular processes. Heterozygous animals (Het) for this protein fed with HFD are more sensitive to insulin than control animals. It is known that the microsomal triglyceride transfer protein (MTP) is a key enzyme for the hepatic production of triglyceride-rich lipoproteins, such as VLDL. Insulin can regulate VLDL secretion by multiple mechanisms ${ }^{1}$, including modulation of the rate of synthesis and degradation of MTP2. When the insulin pathway is active there is a decrease in MTP gene expression, favoring steatosis, as our model is more sensitive to insulin, this is more severe, preventing the VLDL from being secreted into the circulation. The aims of our study were to investigate the participation of ARHGAP21 in the formation of VLDL vesicles and in the modulation of lipogenesis in the liver of ARHGAP21-Het mouse.

\section{Results and Discussion}

ARHGAP21-Het mice fed on a HFD are more sensitive to insulin than control animals, fed with the same diet. This result was obtained by kITT. Through AKT-Serine 483 phosphorylation in liver, we observed that our model Het does not become insulin resistance when fed with HFD, like the control animal.

Liver of ARHGAP21-Het mice fed with HFD presented lower MTP gene expression and protein content, assessed by qPCR and Western Blotting respectively, which resulted in decrease in VLDL secretion. When treated with Triton WR Tyloxapol our model secrets less VLDL that the control animal, suggesting that ARHGAP21 can modulate the VLDL secretion.

Also, those mice presented increased GLUT2 gene expression, measured by qPCR, which corroborates with higher triglycerides accumulation and hepatic steatosis, because the excess of glucose that reaches the liver may be converted in lipids, through De novo lipogenesis pathway, increasing the steatotic state.
Image 1: Mechanism of VLDL synthesis ${ }^{3}$

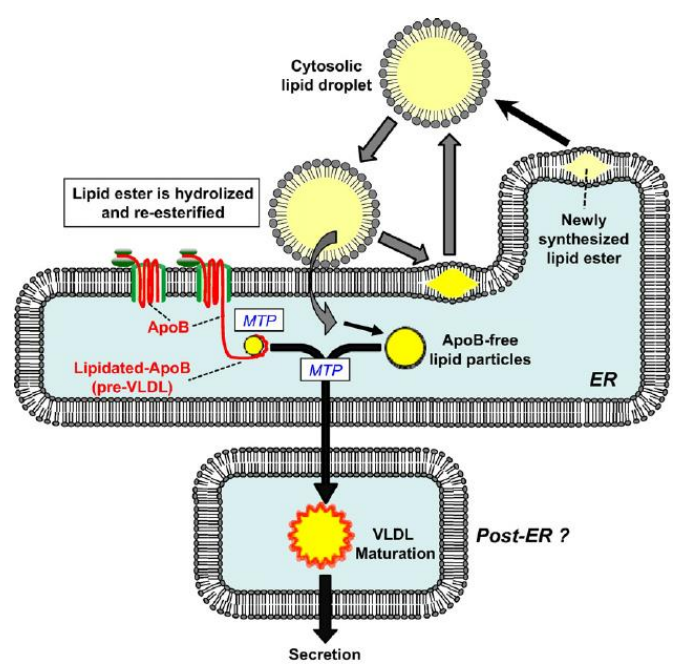

Conclusions

Our results suggest a significant participation of ARHGAP21 on hepatic lipid metabolism, mainly from our data with Het animals treated with a high fat diet (HFD).

\section{Acknowledgement}

FAPESP, OCRC, Biology Institute.

${ }^{1}$ Choi, S. H., et al., Increased very low density lipoprotein (VLDL) secretion, hepatic steatosis, and insulin resistance. Trends Endocrinol Metab. 2011 Sep;22(9):353-63.

2 Hagan, D. L., et al., Transcriptional regulation of human and hamster microsomal triglyceride transfer protein genes - cell type-specific expression and response to metabolic regulators. The Journal of Biological Chemistry, v. 269, p. 28737-28744, 1994.

3 Ohsaki. Y., et al., Biogenesis of cytoplasmic lipid droplets: from the lipid ester globule in the membrane to the visible structure. Biochim Biophys Acta. 2009 Jun;1791(6):399-407. 